\title{
Study on Working Memory Characteristics of Different Nerve Types of College Students
}

\author{
Xinguang $\mathrm{Shi}^{1,2}$ \\ ${ }^{1}$ Applied Technology College of Soochow University, Suzhou,china \\ ${ }^{2}$ School of education of Soochow University,Suzhou,china
}

Keywords: Nerve Types, Working Memory, College Students.

\begin{abstract}
The purpose of this study was to investigate the differences in working memory among different nerve types of college students. A total of 562 college students were tested with $80 \bullet 8$ Nerve Type Test Scale and sorted according to the test scores, then each of the 21 subjects were randomly selected from the $27 \%$ and the last $27 \%$ subjects. 42 participants were required to finish Operation span task and Rotation span task. Results showed that: there was a significant difference in working memory capacity between the high and low score group of the nerve types. Those findings suggest that in contrast to the low score of the nerve types, the high score group has more working memory capacity, faster and higher accuracy processing speed.
\end{abstract}

\section{Introduction}

Some types classified by the basic features of the higher nervous activity in the cerebral cortex are referred as nerve types. Neuro typology is the science of the basic characteristics of the nervous system and the characteristics and laws of difference between individuals. It is a physical and psychological category. Pavlov put forward the theory of advanced neurology through a large number of experimental studies. The theory is that the nervous system has three basic characteristics: the strength, balance and flexibility of the process of excitation and inhibition, and divide people's neural types into different types according to these three basic properties. According to the theory of advanced neurology, considering the developmental level of the cerebral cortex and the law of the activity of the cortical nerve cell excitation and inhibition process, based on the theory of dynamic conditional reflection experiment, Zhang Qinghua and Wang Wenying [1] originally designed a test method of neural type_—"80.8" Nerve Type Test Scale ("80.8" Scale). 80.8 Scale applies to individuals and groups over the age of 6 , it is scientific, objective, and have higher reliability and validity. Since the 1980s, the organization of 27 provinces, autonomous regions and municipalities using the 80.8 scale has worked together to establish a common pattern for the nerve types of the age groups between the ages of 7 and 22 in China. Since then, the Nerve Type Test widely used to select special students, astronauts and athletes, to analyze psychological quality, to analyze individual physiology and mental health, students taught in accordance with their aptitude and so on, and it had a good effect.

Working memory is an energy limited system that stores and processes information temporarily[2], it is the core of human cognitive and advanced cognitive activity, it is also an important component of learning, reasoning, problem solving and intellectual activity[3,4]. In recent years, many studies have found that the training of working memory has a significant effect on the brain's nervous system, the main manifestations are enhanced, attenuated, or rearrangement of nervous system activation[5,6].

Currently, researches on working memory and individual neural activity has been refined into the activity characteristics of individual neuron, dendrites in neurons can receive and store information elements associated with events, and can be transmitted through synapses. Synapses in neurons can be both exciting and reinforcing, and also inhibit the weakening effect. Neurons are linked in 
certain ways, forming the most basic physiological basis of memory. The different properties and types of advanced nervous activity are composed of the intensity of nervous system excitation and inhibition, the balance and flexibility of nervous system activity. Therefore, there may be a strong link between the type of individual nervous system and the working memory.

Although the nerve type and its measurement in many practical applications such as the selection of talents have been widely use, but there is very little research on nerve type and cognitive processing. So far, there is no experimental study on the relationship between nerve type and working memory, this study will explore the relationship between neural activity and working memory, and analyses the differences and causes of individual working memory of different nerve types.

\section{Experiment Methods}

Participants: A total of 562 college students were tested with $80 \bullet 8$ Nerve Type Test Scale and sorted according to the test scores, then each of the 21 subjects were randomly selected from the $27 \%$ and the last $27 \%$ subjects. They aged $18-23$ years old , with a mean age of $21.05 \pm 1.28$ years. All participants reported normal or corrected-to-normal vision, and are right-handed.

Task and Procedure : 80•8 Nerve Type Test Scale. We selected the $80 \bullet 8$ Nerve Type Test Scale by Zhang Qinghua and Wang Wenying. Participants were asked to finish three tasks. Task 1: two symbols of the 8 similar symbols were defined as positive symbols, and the others are negative symbols; Task 2: each line required two symbols to be positive symbols, the others are negative symbols; Task 3: in addition to each line of two symbols for positive symbols, a special symbol was defined as a conditional inhibition symbol, and specified that the positive symbol immediately after the sign became a negative symbol. In three tasks, participants were required to find the positive symbols within 5 minutes as soon as possible. Each 5 minutes' task was recorded by a stopwatch, interval of 5 minutes, totally about 30 minutes. Firstly, we needed to find out the number of total, missing, wrong and planned symbols, then calculated the weighted average score, weighted average error percentage and weighted average leakage percentage, finally, we assessed the total score and nerve type according to the standard.

Operation Span Task. Participants were asked to solve a series of calculation questions, at the same time, they must remember the irrelevant English letters in order of presentation[7]. At the beginning of each trial, mathematical formula was presented to participants, e.g.: $(6+7)-8$, then a number was presented in the center of the screen; Participants were asked to judge whether the number was the result of the formula within 5000 ms. If it timed out, it was judged as error. Then, the center of the screen randomly presented an uppercase letter, e.g.: F, time was $200 \mathrm{~ms}$, participants were required to remember this letter, then the procedure went to the next loop. In each trial, the number of " formula- letter" loops was different, the memory span of the letters was 3-9, each span condition had 3 trail. After the end of each trail, participants were asked to recall subtitles in order. The procedure totally had 126 letters and 126 mathematical formulas. The score of calculation span was the number of letters correctly recalled. In the experiment, the correct rate of judgment task should not be less than $80 \%$. Before the formal experiment, the participants were required to practice the task and the correct rate of practice must be higher than $90 \%$.

Rotation Span Task. This task needed participants to recall a series of long, short arrows and their direction, and finish the judgment of the letter rotation[8]. Firstly, a normal or mirrored rotating letter was presented, the rotation angle was $0,45,90,135,180,225,270$ or 315, participants were asked to judge whether the letter was positive or negative. After the letter judgment, eight long, short arrows pointing to different directions were presented, participants were asked to recall The direction and length of the arrow according to the presentation order. The rotation memory span was 2-6, each span condition had 3 trail. The procedure totally had 60 letters and 60 arrows. The score of rotation span was the number of the direction and length of the arrow correctly recalled. 
Before the formal experiment, the participants were required to practice the task. If the accuracy of practice task was less than $85 \%$, the procedure was automatically terminated.

\section{Results}

After filtering the data other than three standard deviations(a participant in the high group did not complete all the tasks). Scores of two groups in the complex span test were analyzed and found that: Operation Span $(\mathrm{t}=9.07, \mathrm{p}<.001, \eta 2=0.67)$, Symmetry span $(\mathrm{t}=8.27, \mathrm{p}<.001, \eta 2=0.63)$ 。 This result showed that there was a significant difference about the capacity of working memory between the high and low score group of the nerve types. In contrast to the participants getting low scores, the participants getting high scores of the nerve types have more working memory capacity.

Tab.1. Scores of two groups in the Complex Span Test

\begin{tabular}{cccc}
\hline Group & Calculation span & Symmetry span & Participants \\
\hline High score group & $101 . \pm 10.06$ & $51.80 \pm 5.04$ & 20 \\
Low score group & $62.3 \pm 16.77$ & $35.38 \pm 7.49$ & 21
\end{tabular}

Further analysis showed that there was a significant difference in the processing time and processing accuracy between the two groups in the Operation Span Task(Processing time: $\mathrm{t}=$ -2.32, $\mathrm{p}<.05, \eta 2=0.12$; Processing accuracy: $\mathrm{t}=4.71, \mathrm{p}<.001, \eta 2=0.36$ ). The finding suggests that in contrast to the low score group, the processing time of the complex task of the high score group was faster, and the processing accuracy was higher. In other words, the tasks of high score group can be done quickly and accurately.

Tab.2. The processing time and processing accuracy of two groups in the Operation Span Task

\begin{tabular}{llll}
\hline Group & Calculation span & Symmetry span & Participants \\
\hline High score group & $3.04 \pm 0.33$ & $93.76 \pm 4.44$ & 20 \\
Low score group & $3.29 \pm 0.36$ & $86.37 \pm 5.53$ & 21 \\
\hline
\end{tabular}

\section{Discussion}

Before you begin to format your paper, first write and save the content as a separate text file. Keep your text and graphic files separate until after the text has been formatted and styled. Do not use hard tabs, and limit use of hard returns to only one return at the end of a paragraph. Do not add any kind of pagination anywhere in the paper. Do not number text heads-the template will do that for you.

This study found that, there were significant differences in the capacity of working memory for individuals with different nerve types, in other word, in contrast to students in the low score group of the nerve types, subjects in the high score group of the nerve types has more working memory capacity. The results were consistent with the idea that the better the ability of remembering, the more concentrated the individuals were. It supports the analysis of individual cognitive differences in different nerve types [1].

The subjects who scored high on the nerve test were found to be strong and concentrated in the nervous system excitation and inhibition process, the ability of balance is good, the ability of mobility is high, the ability of cortical cells is good and can withstand strong stimulation. Such individuals have faster speed and higher accuracy in encoding, storing and processing external stimuli. In the face of working memory span task with the same cognitive load, the processing of high score group took less time and has higher accuracy, so there is more time for information preservation and retrieval, and the results of working memory span task are better.

The resource sharing model for time[9] accounts that, the cognitive resources used for 
information processing and storage are limited, so resources need to be shared between processing and storage, and as long as the attention is removed from the project, the memory traces of the recalling project will be diminished by time. The amount of time spent on processing is less and more accurate, so more resources are stored. Besides, the processing and operation of high score group took less time, therefore, the degree of extinction of target information by time is low, the speed of extracting information is faster and the attendant resources is less required, so working memory capacity is higher.

The subjects who scored low on the nerve test were weak in the nervous system excitation and inhibition process, the ability of balance is poor, the ability of mobility is low, the ability of cortical cells is weak, so, such individuals have slower speed and poorer accuracy in encoding and processing external stimuli, the processes need more attendant resources, therefore, the attendant resources to encode and keep information are relatively small, the results of storing and keeping of working memory span task are lower. Due to the processing of high score group took more time, the memory traces of the target project will be diminished more by time. The attendant resources of extracting target information is required more, so the results of working memory task is weaker.

\section{Conclusion}

There is a significant difference on the tasks of working memory between the high and low score group of the nerve types . Participants with high neurological scores in working memory capacity was significantly higher than participants with low neurological scores. Taken together, in contrast to the low score group of the nerve types, the high score group of the nerve types has more working memory capacity, faster and higher accuracy processing speed.

\section{Acknowledgement}

In this paper, the research was sponsored by the Project of philosophy and social science fund of University of Jiangsu Provincial Department of Education ～(Project No. 2015SJB542).

\section{References}

[1] Zhang Qinghua, \& Wang Wenying. (1993). Research on the evaluation of human neural types. Beijing: Higher Education Press.

[2]Baddeley,A.D.(2003). Working memory: Looking back and looking forward.Neuroscience,4(10),829-839.

[3]Camos,V.(2008). Low working memory capacity impedes both efficiency and learning of number transcoding inchildren.Journal of Experimental Child Psychology,99,37 -57.

[4]Schecklmann,M., $\quad$ Romanos,M., $\quad$ Bretscher,F., $\quad$ Plichta,M.M., $\quad$ Warnke,A., \& Fallgatter,A.J.(2010).Prefrontal oxygenation during working memory in ADHD. Journalof Psychiatric Research, 44, 621- 628.

[5]Schneiders,J.A.,Opitz,B., Krick,C.M., \& Mecklinger,A.(2011).Separating intra-modal and across-modal training effects in visual working memory: An fMRI investigation. CerebralCortex, 21,2555-2564.

[6]Jolles,D.D., Grol,M.J., van Buchem,M.A., Rombouts,S.A.R.B., \& Crone,E.A.(2010).Practice effects in the brain: Changes in cerebral activation after working memory practice depend on task demands. Neuroimage, 52, 658-668. 
[7]Unsworth,N.(2010a).Interference control, working memory capacity, and cognitive abilities: A latent variable analysis. Intelligence, 38, 255- 267.

[8]Wilhelm,O.,\& Oberauer,K.(2006).Why are reasoning ability and working memory related to mental speed? An investigation of stimulus-response compatibility in choice reaction time task.European Journal of cognitive Psychology, 18, 18-50.

[9]Pierre,B., Sophie,B., \& Valene,C.(2004). Time Constraints and Resource Sharing in Adult's Working Memory Spans. Journal of Experimental Psychology: general, 133, 83-100. 